\title{
All-inorganic halide-perovskite-polymer luminescent fibers for high-bitrate ultraviolet free-space optical communication
}

\author{
Chun Hong Kang ${ }^{1}$, Omar Alkhazragi ${ }^{1}$, Lutfan Sinatra ${ }^{2}$, Sultan Alshaibani ${ }^{1}$, Kuang-Hui Li ${ }^{1}$, Meiwei Kong ${ }^{1}$, Marat \\ Lutfullin $^{2}$, Osman M. Bakr ${ }^{3}$, Tien Khee $\mathrm{Ng}^{1}$, and Boon S. Ooi ${ }^{1, *}$ \\ ${ }^{1}$ Photonics Laboratory, Division of Computer, Electrical, and Mathematical Sciences and Engineering and ${ }^{3}$ Division of Physical \\ Science and Engineering, King Abdullah University of Science and Technology (KAUST), Thuwal 23955-6900, Saudi Arabia \\ ${ }^{2}$ Quantum Solutions, 1 Venture Road, Science Park, Southampton, SO16 7NP, United Kingdom \\ *Email address: boon.ooi@kaust.edu.sa
}

\begin{abstract}
We demonstrate, for the first time, halide-perovskite-polymer-based luminescent fibers as a high-bitrate and near-omnidirectional photodetection platform for 375-nm ultraviolet laser-based free-space optical communication. Our demonstrations could enable future ultraviolet-to-visible optical receivers based on all-inorganic halide-perovskite nanomaterial to cater various dynamic scenarios in optical wireless communication.
\end{abstract}

Keywords-perovskite luminescent fiber, optical detectors, free-space optical communication

\section{INTRODUCTION}

In recent years, the previously uncharted ultraviolet (UV)-based free space optical wireless communication, i.e., $<380 \mathrm{~nm}$, has received significant attention due to its higher Rayleigh scattering as compared to the visible light, that eventually leads to various demonstrations and realization of non-line-of-sight (NLOS) or diffuse-line-of sight (diffuseLOS) modalities on both terrestrial and underwater settings [1]. While there is considerable attention on the development of UV transmitters, the receiver end suffers from various challenges in terms of the costly and timeconsuming development path of large bandgap materials (i.e., III-nitride or III-oxide), and could hinder the efforts for exploring additional channel capacity beyond the visible range. Due to the high atmospheric scattering nature of the UV light, the receivers are expected to be of large field of view (i.e., omnidirectional), high modulation bandwidth, and large detection area in order to effectively capture the incoming optical signals. Luminescent fibers, utilizing the wavelength-conversion and waveguiding methods to effectively propagate the received signals, were first conceived as a high-speed optical detection platform by Peyronel et al. [2]. This method offers high optical gain that scales with the number and size of luminescent fibers, as well as eliminating the conventional tradeoff between detection area and modulation bandwidth (i.e., resistance-capacitance limit) in planar-based photodiodes, while featuring a wide field of view. While several prior works highlighted on organic dye-based luminescent fibers or concentrators, in this work, we shed light on integrating an all-inorganic-based luminescent dye using $\mathrm{CsPbBr}_{3}$ nanocrystals with a higher conversion efficiency as compared to the organic counterparts, to form a perovskite-polymer luminescent fibers-based detection platform. Our first proof-of-concept demonstration opens up a new uncharted field of applications of halide-perovskitebased materials beyond the conventional light-emitting diodes or solar cell applications.

\section{RESUlTS AND DISCUSSION}

The schematic diagram in Fig. 1(a) illustrates the fundamental concept of luminescent fibers presented herein for high-speed optical wireless communication systems, where the luminescent dye doped within the core section of the polymer fiber emits the light signal upon an optical excitation through the cladding layer, which then propagates through total internal reflection (TIR) towards the fiber ends. The optical signals coupled out from the fibers array could be guided into a high-speed photodiode for optical-to-electrical (O-E) conversion and data demodulation. Figure 1(b) shows the transmission electron microscopy (TEM) image of the all-inorganic $\mathrm{Cs}_{\mathrm{PbBr}}$ nanocrystals ( $\mathrm{NCs}$ ) used in this work, where the nanocrystals are cubic shaped with an average edge length of approximately $6.4 \mathrm{~nm}$, as well as the photoluminescence (PL) peak wavelength in the vicinity of $\sim 515 \mathrm{~nm}$ (see the inset of Fig. 1(b)). The PL quantum yield of the sample was measured to be more than $95 \%$ and was significantly higher as compared to some other organicbased dyes, e.g. SuperYellow (60\%) and Coumarin-6 (80 to 95\%).

Figure 1(c) shows a photograph image of a perovskite-polymer-based luminescent fiber, having the composite of $\mathrm{CsPbBr}_{3} \mathrm{NCs}$ and isobornyl acylate (IBOA) forming the core section with a refractive index $(n)$ of 1.476, while the cladding layer consists of polydimethylsiloxane (PDMS) with $n$ of 1.436 . The core layer formed by $\mathrm{CsPbBr}_{3} \mathrm{NCs}-$ IBOA mixture was fully cured under a 365-nm light source. As shown in Fig. 1(d), the core layer made of $\mathrm{CsPbBr}_{3}$ NCs-IBOA with an optimized NCs concentration of $2 \mathrm{mg} / \mathrm{mL}$ is about $950 \mu \mathrm{m}$ in diameter. Conceived as a high-speed optical detection platform based on the proposed $\mathrm{CsPbBr}_{3} \mathrm{NCs}$-polymer luminescent fiber, the radiative recombination 
lifetime was also characterized to be $\sim 2.96 \mathrm{~ns}$, elucidating its potential as a high-speed conversion material [3]. Figure 1(e) further shows the modulation bandwidth of the perovskite-polymer luminescent fibers-based receiver measured using a 375-nm UV laser diode, where the average -3-dB bandwidth $\left(f_{-3 \mathrm{~dB}}\right)$ is approximately $12.34 \mathrm{MHz}$ for a $30-\mathrm{mm}$ long luminescent fiber, limited by the radiative recombination lifetime of the perovskite NCs, thereby circumventing the resistance-capacitance limit in planar detectors. The bit error ratio (BER) versus data rate plot, shown in Fig. 1(f), demonstrates that the perovskite-polymer scintillating luminescent fibers array (see inset of Fig. 1(f)) is able to support up to $20 \mathrm{Mbit} / \mathrm{s}$, with BER below the forward-error correction (FEC) limit, based on non-return-to-zero on-off keying (NRZ-OOK) modulation scheme in a 375-nm UV-based transmission channel.
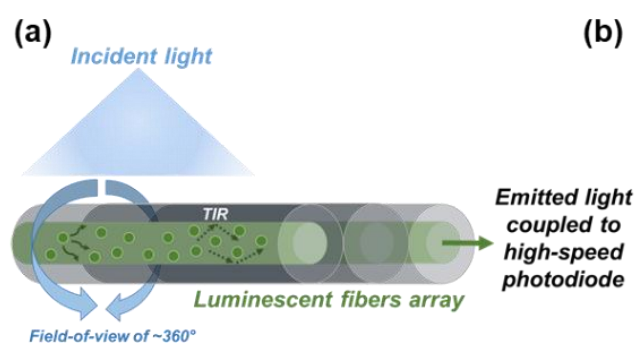

(d)

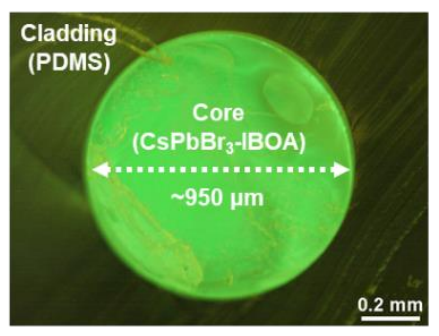

(b)

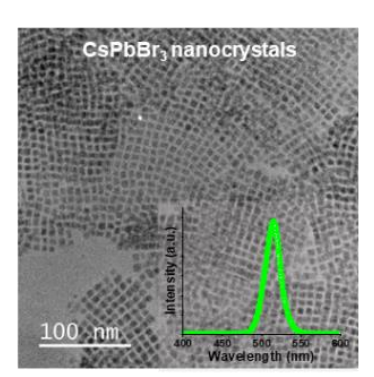

(e)

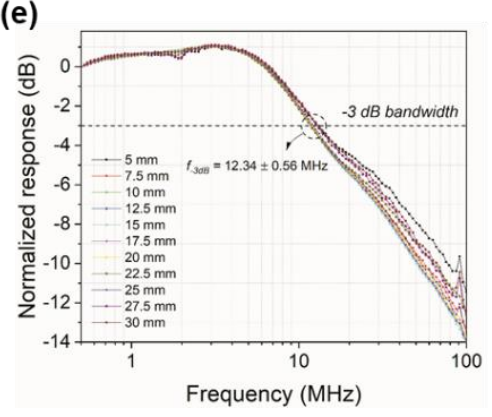

(c)

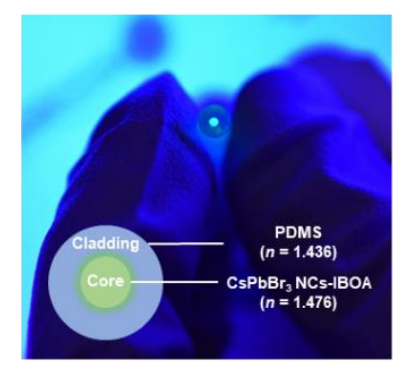

(f)

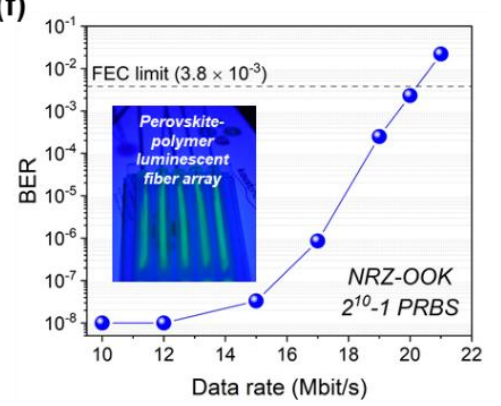

Fig. 1 (a) Schematic illustration of the concept of luminescent fibers-based optical receiver. (b) Transmission electron microscopy (TEM) image of the $\mathrm{CsPbBr}_{3}$ nanocrystals. The inset shows the corresponding photoluminescence spectrum under $405-\mathrm{nm}$ excitation. (c) Photograph image of the perovskite-polymer luminescent fibers end under UVC illumination. (d) Zoomed-in image of the perovskite-polymer luminescent fiber, where the core layer is formed by $\mathrm{CsPbr}_{3}$-IBOA mixture and the cladding layer is made of PDMS. (e) Modulation bandwidth of the perovskite-polymer luminescent fiber measured across a 30-mm-long fiber. (f) Bit-error ratio versus data rate based on NRZ-OOK modulation scheme with a 375-nm laser diode as transmitter and the proposed perovskite-polymer luminescent fiber array as the receiver platform.

\section{CONCLUSION}

In this work, we demonstrated perovskite-polymer luminescent fibers as a high-speed optical detection platform for up to $20 \mathrm{Mbit} / \mathrm{s}$ UV-based free-space optical communication. Owing to the short transition lifetime, high photoluminescence quantum yield of the $\mathrm{CsPBBr}_{3}-\mathrm{IBOA}$ composite, as well as the inherent nature of luminescent fibers enabling large-area, wide-field-of-view optical detection platform, our first-of-its-kind approach based upon wavelength-tunable perovskite nanocrystals sheds light on new avenues for perovskite-based optoelectronic applications, while obviating the costly and time-consuming development path of other large bandgap compound semiconductors as a high-speed optical detection platform

\section{REFERENCES}

[1] A. Vavoulas, H. G. Sandalidis, N. D. Chatzidiamantis, Z. Xu, and G. K. Karagiannidis, "A Survey on Ultraviolet C-Band (UV-C) Communications," IEEE Commun. Surv. Tutorials, vol. 21, no. 3, pp. 2111-2133, 2019.

[2] T. Peyronel, K. J. Quirk, S. C. Wang, and T. G. Tiecke, "Luminescent detector for free-space optical communication," Optica, vol. 3, no. 7, pp. 787-792, 2016.

[3] C. H. Kang, I. Dursun, G. Liu, L. Sinatra, X. Sun, M. Kong, J. Pan, P. Maity, E.-N. Ooi, T. K. Ng, O. F. Mohammed, O. M. Bakr, and B. S. Ooi, "High-speed colour-converting photodetector with all-inorganic $\mathrm{CsPbBr} 3$ perovskite nanocrystals for ultraviolet light communication," Light Sci. Appl., vol. 8, no. 1, p. 94, 2019.

\section{ACKNOWLEDGMENT}

The authors acknowledge funding support from the U.S. Office of Naval Research Global (ONRG) under award number N62909-19-1-2079 (KAUST reference number: RGC/3/4119-01-01) and King Abdullah University of Science and Technology (KAUST) (Grant No. BAS/1/1614-01-01, KCR/1/2081-01-01, and GEN/1/6607-01-01). T. K. N. and B. S. O. acknowledge the support from King Abdulaziz City for Science and Technology (KACST) (Grant No. KACST TIC R2-FP-008). 